\title{
Immunoglobulin profile of the preterm baby
}

\author{
S P CONWAY, P R F DEAR, AND I SMITH \\ Department of Paediatrics and Child Health, St James's University Hospital, Leeds
}

SUMMARY Immunoglobulin concentrations were determined in 64 consecutively born preterm babies at birth and serially throughout each baby's stay in the neonatal unit. No significant IgG generation was found during the first 15 weeks of life, regression analysis giving an exponential decay model. Concentrations fell to $2 \mathrm{~g} / \mathrm{l}$ or less in $10(16 \%)$ babies (gestational age 25 to 32 weeks), and were as low as $1 \mathrm{~g} / \mathrm{l}$ in four babies (gestational age 25 to 29 weeks). The effects of gestational age and birthweight on the concentration of IgG at birth were highly interdependent and significant. Most babies had no detectable $\operatorname{IgA}$ at birth, and no effect of gestational age or birthweight, or both, on either initial IgA or IgM concentrations could be shown.

Infection is a frequently encountered problem among preterm infants, whose defences against invasion by micro-organisms are limited in several respects. In terms of the humoral immune system, the preterm neonate is compromised at birth by having lower concentrations of IgG than his term counterpart, neonatal IgG being largely of maternal origin $^{1}$ and transferred mostly as the fetus nears term. ${ }^{2} \mathrm{He}$ may also be deficient in endogenous immunoglobulin production. ${ }^{3}$ These factors may combine to produce a state of pathological hypogammaglobulinaemia with an associated increased susceptibility to infection. ${ }^{4}$

Although there has been extensive research into the immunological status of the newborn, there is little data on the immunoglobulin profile of the preterm infant, and to our knowledge no serial studies that include many extremely low birthweight infants. The purpose of the present study was to document the pattern of serum immunoglobulin concentrations in the preterm neonate throughout his stay on the neonatal unit, to attempt to relate the findings to the incidence of infection, and to investigate the effect, if any, of replacement blood transfusions.

\section{Patients and methods}

The study population was 64 babies of less than 36 weeks' gestation admitted consecutively to the neonatal unit. Gestational age, determined from ultrasound scanning at 17 weeks' gestation, or less, or by clinical assessment, ${ }^{5}$ ranged from 25 to 36 weeks (mean 32 weeks) and birthweight from 0.65 to $2.7 \mathrm{~kg}$ (mean $1.65 \mathrm{~kg}$ ). Immunoglobulin values were determined by single radial immunodiffusion. Low level plates were used for all samples, except in eight samples of IgM and five of IgA. Initial immunoglobulin concentrations were determined within an hour of birth where possible, and always within the first 24 hours and before transfusion of any blood or other products. Serial serum immunoglobulin values were assayed thereafter at weekly intervals until discharge from the unit. The samples were drawn concurrently with routine haemoglobin samples.

On clinical suspicion of infection, full blood count, blood and urine culture, chest radiograph, swabs, and in most cases cerebrospinal fluid culture were investigated.

All blood removed and all blood and blood products transfused were noted.

\section{Results}

Immunoglobulin status at birth. Sixty two values for IgG, 54 for IgM, and 57 for IgA were obtained at birth from the 64 babies included in the study.

IgG values at birth ranged from $2.6 \mathrm{~g} / \mathrm{l}$ to $14.4 \mathrm{~g} / \mathrm{l}$ (median $6.7 \mathrm{~g} / \mathrm{l}$ ). Multiple regression analysis using the values of IgG, gestational age, and birthweight showed that the concentration of IgG at birth was affected by gestational age or birthweight, or both, and also indicated that the residual variation in IgG increased with the IgG value, suggesting that a better analysis is obtained using $\log _{e}(\mathrm{IgG})$ as the response variable. The most comprehensive mathematical model relating the variables concerned is: 
$\mathrm{X}_{2}$

$$
\log _{e}(I g G)=2 \cdot 084+0 \cdot 1240 X_{1}+2 \cdot 01 X_{2}-0 \cdot 0535 X_{1}
$$

which can be written in the form:

$\log _{e}(\operatorname{IgG})=2 \cdot 084-0.0535\left(X_{1}-37 \cdot 58\right)\left(X_{2}-2 \cdot 318\right)$ where $X_{1}$ is gestational age and $X_{2}$ is birthweight. This model accounts for $46 \%$ of the total variation in $\log _{e}(\mathrm{IgG})$ and is significant at the $1 \%$ level. It is shown graphically in Fig. 1. The precise form of the relation is somewhat obscured by the high correlation between gestational age, $\left(X_{1}\right)$ and birthweight $\left(\mathrm{X}_{2}\right)$.

A distinctive feature of the model is its implication that the rate of increase of $\log _{e}(\operatorname{IgG})$ with birthweight is highest at low gestational ages and becomes negligible at gestational ages of 34 weeks and above.

The 54 values of IgM at birth are shown in Fig. 2 . There was no detectable effect of gestational age or birthweight on the concentration of IgM at birth.

IgA was found in only 10 of 57 samples taken at birth. The gestational ages and birthweights of the 47 babies with no detectable $\operatorname{IgA}$ and the 10 babies in whom this was found were randomly spread over the data. The 10 measured values of $\operatorname{IgA}$ ranged from 0.02 to $0.48 \mathrm{~g} / \mathrm{l}$, (median $0.22 \mathrm{~g} / \mathrm{l}$ ): this variation was not accounted for by either gestational age or birthweight, or both.

Immunoglobulin status during the first 15 weeks of life. IgG was measured at weekly intervals for up to 15 weeks in 51 of the babies - a total of 313 observations. On the same weekly basis, measure-

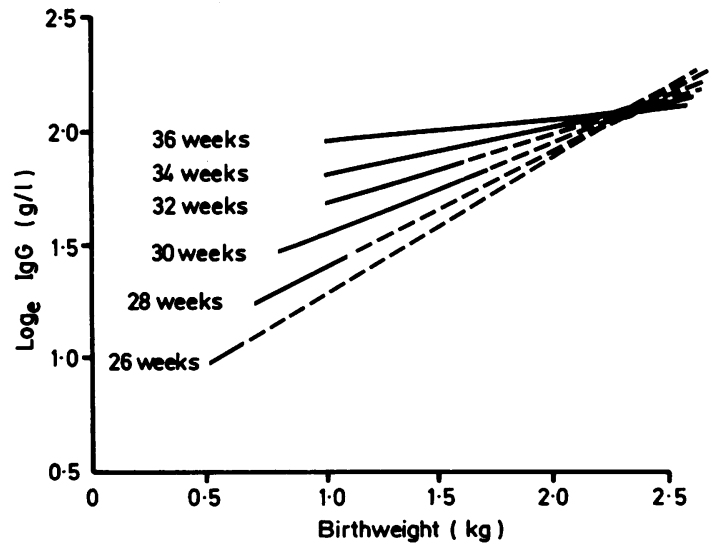

Fig. $1 \operatorname{IgG}\left(\log _{e} g / l\right)$ against birthweight $\left(X_{2}\right)$ and gestational age $\left(X_{l}\right)$.

\footnotetext{
The regression equation:

$\log _{c}(\mathrm{IgG})=2 \cdot 084-0 \cdot 0535\left(\mathrm{X}_{1}-37 \cdot 58\right)\left(\mathrm{X}_{2}-2 \cdot 318\right)$ is illustrated by $\log _{c}$ (IgG) $v$ birthweight at stated gestational ages, with the solid lines covering the range of data studied.
}

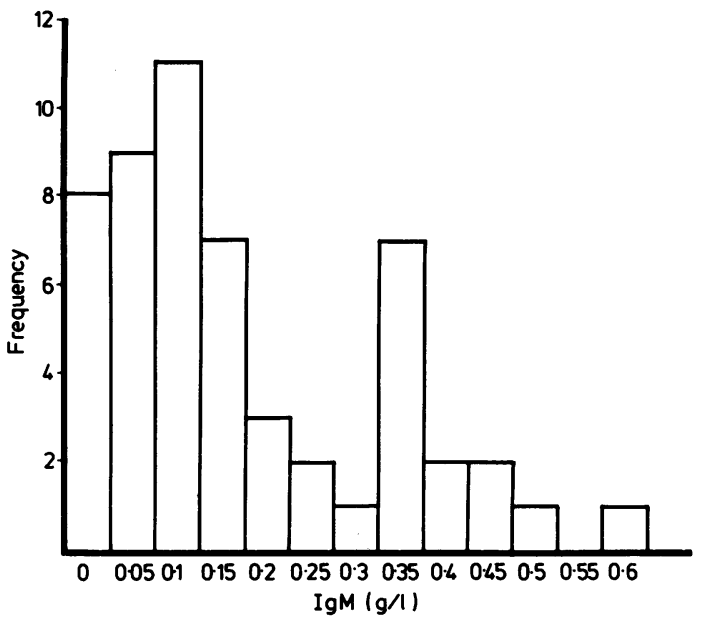

Fig. 2 Frequency distribution of values of $\operatorname{IgM}$ at birth.

ments were also made of weight $(\mathrm{kg})$, number of infections, amount of blood transfused $(\mathrm{ml})$, and amount of blood withdrawn ( $\mathrm{ml}$ ).

Overall, the graph of IgG against age shows that IgG declines as age increases (Fig. 3). Regression analysis indicates that the best-fitting equation is the exponential decay model $\log _{e}(I g G)=a_{n}-0.0898$ age, where $a_{n}$ is a constant for the $n^{\text {th }}$ baby. The regression coefficient for age is highly significant at the $1 \%$ level. Serum IgG values fell to $1.0 \mathrm{~g} / \mathrm{l}$ in four of seven babies of 29 weeks' gestation or less, and

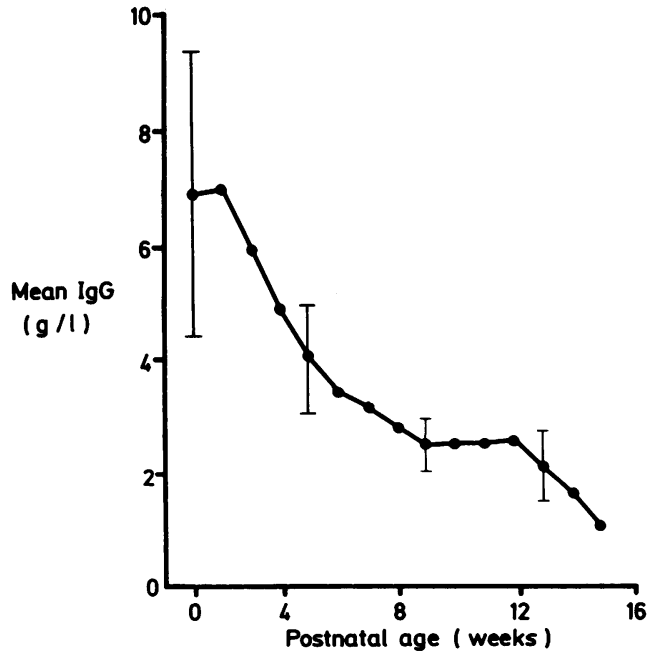

Fig. 3 Change in $\operatorname{Ig} G$ value with age.

Regression equation $\log _{c}(I g G)=a_{n}-0.0898$ age. $(I=I S D)$ 
concentrations of $2.0 \mathrm{~g} / \mathrm{l}$ or less were recorded in 10 $(16 \%)$ of the babies. Part of the variation from one baby to another may be accounted for by the effect of birthweight, the inclusion of which gives the regression equation, $\log _{e}(\operatorname{IgG}),=b_{n}-0.0898$ age + 0.502 birthweight; where $b_{n}$ is a constant for the $n^{\text {th }}$ baby, for which the regression coefficient for birthweight is significant at the $1 \%$ level.

The effect on IgG of postnatal weight gain was studied in terms of the model form;

IgG $\times$ weight $->$ constant

In regression analysis terms the following variables were considered:

$\mathrm{y}=\log _{\mathrm{e}}(\mathrm{IgG} / \mathrm{IgG}$ at birth $)$;

$\mathrm{X}_{1}=\log _{\mathrm{e}}$ (weight/birthweight);

$\mathrm{X}_{2}=$ age in weeks;

191 observations of this data were available for 24 babies.

The graphs of $y$ against $X_{1}$ (Fig. 4) and $y$ against $X_{2}$ (Fig. 5) both show clear relations, but they are not independent because of the high correlation between $X_{1}$ and $X_{2}$ (Fig. 6).

The regression of $y$ on $X_{1}$ gives the equation $y=0 \cdot 0914-1 \cdot 10 X_{1}$ with both coefficients significant. The coefficient of $X_{1}$ is, moreover, not significantly different from -1 , thus supporting the hypothesis that IgG $\times$ weight is approximately constant. Further analysis indicated that, for practical purposes, the change in IgG with age is attributable to corresponding changes in weight. In fact the quantity

$$
\frac{(\operatorname{IgG} \times \text { weight })}{\text { initial }(\operatorname{IgG} \times \text { weight })}
$$

does not change significantly with age in the first 15 weeks, indicating that there is little or no IgG generation during this time.

There were 40 separate clinically suspected episodes of infection in the 64 babies, 36 of these occurring in babies of 30 weeks' gestation or less. In

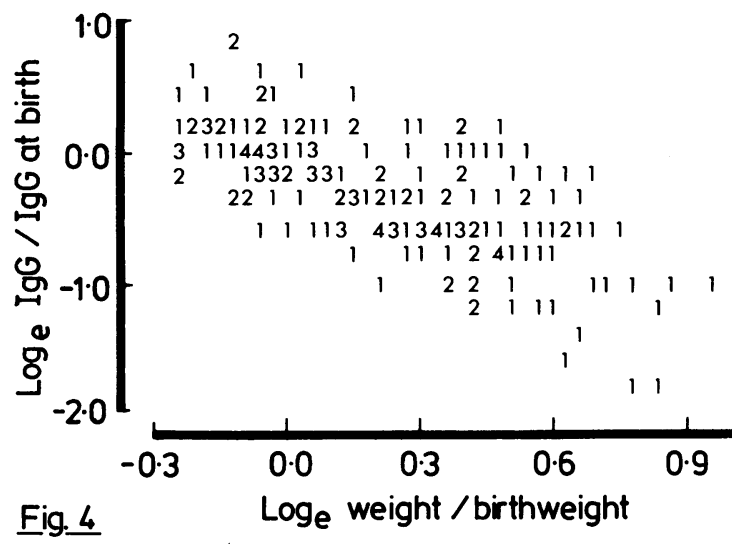

Fig. $4 \quad \log _{\mathrm{e}}$ weight /birthweight

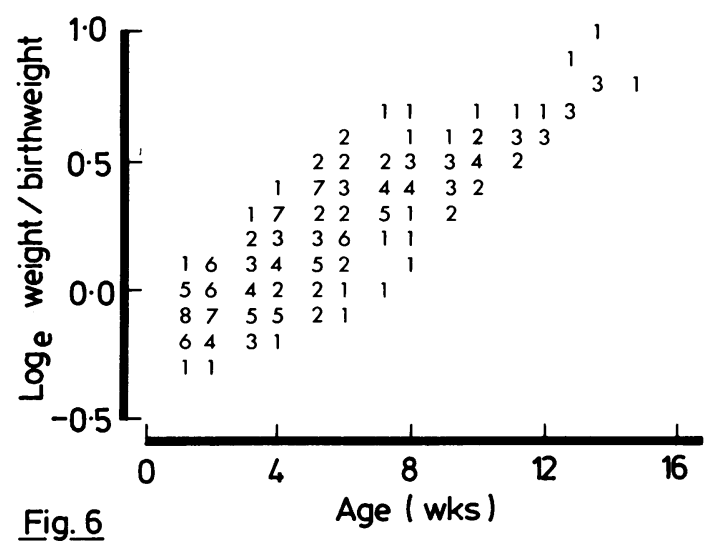

Fig. 4 Change in IgG value against change in weight.

Fig. 5 Change in $\operatorname{Ig} G$ value against postnatal age.

Fig. 6 Change in weight against postnatal age. 
13 of the 40 episodes, a positive blood culture was obtained: Gram negative organisms were isolated on five occasions and Staphylococcus albus on eight. In five of the 27 infective episodes without positive blood culture, the infection was thought to be predominantly respiratory. There was no case of urine infection or meningitis. No significant effect of the variables of infections, or blood transfusion, or blood withdrawals on IgG was found. No definite association was found between absolute IgG concentrations and the incidence of presumed or proved infection.

A total of 308 observations of serial IgM values were made on 51 babies. The regression of IgM on age (in weeks) gave the equation, $\mathrm{IgM}=0 \cdot 271+0 \cdot 121$ age $-0.0076(\text { age })^{2}$. The regression coefficients were highly significant. The implication of this equation is that $\mathrm{IgM}$ rises from an average value of $0.27 \mathrm{~g} / \mathrm{l}$ at birth to a peak of $0.75 \mathrm{~g} / \mathrm{l}$ after 8 weeks. Analysis of postnatal IgA values showed no reproducible pattern.

\section{Discussion}

The immunoglobulin concentrations at birth in this study agree with the earlier work of $\mathrm{Hobbs}^{4}$ and Yeung, ${ }^{6}$ confirming the linear relation between the logarithm of the initial IgG concentration and gestational age. Figure 1 shows that the effect of gestational age is greatest at low birthweights (less than $1 \mathrm{~kg}$ ) and reduces progressively until it becomes negligible at birthweights of $2 \mathrm{~kg}$ and above.

The high correlation between gestational age and birthweight tends to obscure the identification of their separate effects, though others have considered birthweight to be an important influence on the initial concentration of $\mathrm{IgG}$. Yeung ${ }^{6}$ and Papadatos $^{7}$ showed that small for dates babies commonly have an IgG concentration less than the mean for gestational age. The small for dates variable, however, is derived from gestational age and birthweight and we felt that it should not really be considered as an independent variable. In practice it was never found to exert an independent influence, and it is not discussed further.

It is an established fact that transplacentally acquired IgG constitutes most of that present at birth, and that as this decays and is diluted by an increasing blood volume, serum IgG concentrations fall until overtaken by endogenous IgG synthesis. Figures 4 and 5 clearly illustrate this fall in serum IgG with weight gain and with increasing age to at least 15 weeks. The data showed no sign that the fall in serum IgG was remitting, other than exponentially, at the end of the study period.
The baby's defence mechanisms against infection are complex, humoral immunity being but a part, and we accept that this study shows a low biochemical IgG value and not a functional deficit. Other factors are also likely to be important in protecting the baby against infection.

The passive transfer of immunity in the form of secretory $\operatorname{IgA}$, the predominant immunoglobulin in human milk, may be particularly important to the preterm baby, whose local intestinal immunity is incompletely developed. Secretory $\operatorname{IgA}$ is at its highest concentration in colostrum, and at a significantly higher concentration in milk from mothers giving birth preterm. ${ }^{8}$ Only eight $(32 \%)$ of 25 babies of 32 weeks' gestation or less were established on human milk feeds within the first month, and all received banked breast milk.

The ability to produce high concentrations of secretory $\operatorname{IgA}$ in response to acute respiratory tract infections protects against recurrent infection in children as young as 6 months, ${ }^{9}$ and may be important in the preterm baby. Human milk may also transfer specific immunity to the respiratory tract. ${ }^{10}$

Five of 13 positive blood cultures grew Gram negative organisms, infections in which complement and neutrophil defects may play an important part.

Cord blood IgM concentrations may be raised in intrauterine infection. ${ }^{1518} 19$ Our data showed no significant relation between serial postnatal IgM values and episodes of infection. This does not, however, take account of the importance of functional IgM antibodies.

Occasional, low IgG concentrations have been documented in apparently healthy children aged 6 months to 1 year, ${ }^{11}$ and test immunisation of patients with primary hypogammaglobulinaemia has also shown little antibody response, despite their remaining in relatively good health. ${ }^{12}$ Nonetheless, this study clearly shows that serum IgG concentrations in preterm, low birthweight babies may fall to values which in absolute terms can only be seen as dangerously low. Moreover, the IgG values documented in our study are not seen in the term baby, in whom antigenic stimulation results in increased immunoglobulin synthesis after birth as the immune system matures. It seems likely that the exposure of the babies in our study to varied antigens was at least as extensive as that of any mature infant and we speculate that there is a deficit in the acquisition of humoral immunity in the least mature babies. With the improving survival prospects of the very preterm baby, we believe that the possibility that the low concentrations of IgG shown may increase the risk of infection appreciably warrants more extensive investigation, and that the 
place for prophylactic injection of immune gammaglobulin merits consideration and study.

Work in this area to date has produced conflicting results. Hobbs ${ }^{4}$ and $\mathrm{Amer}^{13}$ both suggest that a single injection of human gammaglobulin in the first week of life may confer some protection against subsequent infection. In contrast, Steen ${ }^{14}$ found no decreased infection risk in preterm babies given prophylactic gammaglobulin at intervals during early life, but his numbers were small and he conceded that both the dosage and the dosage interval may need reassessment.

Analysis of the data obtained on $\operatorname{IgA}$ and $\operatorname{IgM}$ values at birth suggests that neither reflects birthweight or gestational age. This is in agreement with most other authors, ${ }^{2}{ }^{315-17}$ as is our finding of negligible concentrations of IgA at birth..$^{216-19}$ The data provide no indication of the factors that determine the presence or absence of $\operatorname{IgA}$ at birth, or of its quantity when present.

A rise in IgM to a peak after 8 weeks was observed, but it was not possible to relate this to any particular event in the baby's life. It may merely be a reflection of its being the chief immunoglobulin synthesised by the neonate ${ }^{20}$ or a response to a new microbiological environment. ${ }^{17}$

The study shows how severely immunologically compromised the preterm baby may become. The low birthweight, low gestational age baby is particularly at risk. Neither blood transfused or removed has any significant influence on the steady fall in IgG values during the early weeks of life. No effective IgG synthesis was shown by any of our population.

We thank Dr A Baines for help with the statistical analysis and Dr Swinburne for performing the immunoglobulin measurements.

\section{References}

${ }^{1}$ Bridges RA, Condies RM, Zak SJ, Good RA. The morphological basis of antibody formation development during the neonatal period. J Lab Clin Med 1959;53:331-57.

2 Salimonu LS, Ladipo OA, Adeniran SO, Osukoya BO. Serum immunoglobulin levels in normal, premature and postmature newborns and their mothers. Int J Gynaecol Obstet 1978;16: $119-23$.
${ }^{3}$ Ravivarma KR, Babar ST, Master J, Bapat JP, Baxi AJ. Immunoglobulins in newborns: differential study of premature and full term infants. J Postgrad Med 1979;25:97-101.

4 Hobbs RJ, Davis AJ. Serum gamma G-globulin levels and gestational age in premature babies. Lancet 1967;i:757-9.

5 Dubowitz L, Dubowitz V, Goldberg C. Clinical assessment of gestational age in the newborn infant. $J$ Pediatr 1970;77:1-10.

6 Yeung YC, Hobbs RJ. Serum gamma G globulin levels in normal, premature, postmature, and small-for-dates newborn babies. Lancet 1968; i:1167-70.

7 Papadatos C, Papaevangelou GJ, Alexiou D, Mendris J. Serum immunoglobulin $\mathrm{G}$ levels in small-for-dates newborn babies. Arch Dis Child 1970;45:570-2.

- Gross SJ, Buckley RH, Wakil SS, McAllister DC, David RJ, Faix RG. Elevated IgA concentration in milk produced by mothers delivered of preterm infants. J Pediatr 1981;99:389-93.

9 Yodfat Y, Silvian I. A prospective study of acute respiratory tract infections among children in a kibbutz: the role of secretory IgA and serum immunoglobulins. J Infect Dis 1977; 136:26-30.

${ }^{10}$ Hanson LA, Luinberg J. Breast milk and defence against infection in the newborn. Arch Dis Child 1972;47:845-7.

"Isaccs D, Altman DG, Tidmarsh CE, Valman HB, Webster ADB. Serum immunoglobulin concentrations in preschool children measured by laser nephelometry: reference ranges for IgG, IgA, IgM. J Clin Pathol 1983;36:1193-6.

12 Webster ADB, Latif AAA, Brenner MK, Bird D. Evaluation of test immunisation in the assessment of antibody deficiency syndromes. $\mathrm{Br}$ Med $J$ 1984;288:1864-6.

13 Amer J, Off E, Ibbott FA, O'Brien D, Kempe CH. The effect of monthly gamma-globulin administration on morbidity and mortality from infection in premature infants during the first year of life. Pediatrics 1963;32:4-9.

14 Steen JA. Gamma globulin in preventing infections in premature infants. Archives of Pediatrics 1960;77:291-4.

15 Cederquist LL, Ewool LC, Litwin SD. The effect of fetal age birthweight, and sex on cord blood immunoglobulin values. Am J Obstet Gynecol 1978;131:520-5.

${ }^{16}$ Evans HE, Akpata SO, Glass L. Serum immunoglobulin levels in premature and full term infants. Am J Clin Pathol 1971;56: 416-8.

17 Mahambare AP, Iyer YS, Joshi MK, Irani SF, Kandoth WP. Neonatal immunoglobulins. Indian Pediatr 1978;15:577-81.

18 Papadatos C, Papaevangelou GJ, Alexiou D, Mendris J. Immunoglobulin levels and gestational age. Biol Neonate 1969;14:365-73.

19 Gusden JP. Fetal and maternal immunoglobulin levels during pregnancy. Am J Obstet Gynecol 1969;103:895-900.

20 Stiehmn ER, Fudenberg HH. Serum levels of immunoglobulins in health and disease. A survey. Pediatrics 1966;37:715-27.

Correspondence to Dr S P Conway, University Department of Paediatrics, General Infirmary, Leeds LS2 9NS.

Received 30 November 1984 